\title{
Cinética para recuperación de zinc de cenizas de galvanizado con ácido clorhídrico
}

\section{Kinetics for recovery of zinc from ashes of galvanized with hydrochloric acid}

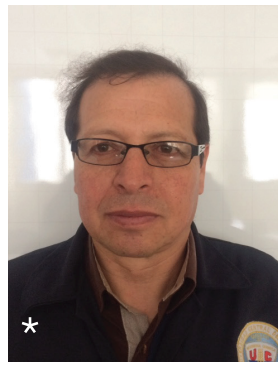

*Mario Calle. Facultad de Ingeniería Química. Universidad de Central de Ecuador / mcalle@uce.edu.ec

Isnel Benítez Cortés, Amaury Pérez Martínez

Departamento de Ingeniería Química, Universidad de Camagüey, Cuba.

Resumen

La extracción del zinc que contienen las cenizas de galvanizado en caliente, se realizó usando ácido clorhídrico. La composición de las cenizas fue determinada por difracción de rayos x, obteniéndose zinc metálico, oxido y el hidróxido, siendo el mayor componente el óxido (49\%). Las cenizas son sólidos granulares de forma irregular, las partículas con un diámetro promedio mayor dio las mayores tasas de velocidad. Las representaciones gráficas del modelo del núcleo que se encoje sin reaccionar controlado por la reacción química dieron una buena linealidad. Así mismo, se demostró que la velocidad de lixiviación no depende de la agitación, y la energía de activación estimada dio un valor de 18,4kj/mol. La velocidad de la reacción puede ser determinada mediante la siguiente ecuación semiempírica:

$$
\left[1-\left(1-X_{B}\right)^{\frac{1}{3}}\right]=\frac{12784 e^{\frac{-18400}{R T}} C_{A L 0}^{1,4686}}{\rho_{B} R_{0}} t+0,1012 ; t \geq 1 \mathrm{~min} .
$$

Palabras clave: cinética; extracción; reacción heterogénea; ácido; cenizas de zinc

\section{Abstract}

The leaching of zinc from galvanic ashes was carried out using hydrochloric acid. Ashes composition includes metal zinc, oxide and hydroxide according to the X-rays diffractrometry, being the greater component the oxide (49\%). The ashes are powders of irregular shape. Powders with smaller mean diameter gave the higher rates. The plots of the shrinking core model controlled by the chemical reaction gave good linearity. Also the leaching rate did not depend on stirring and the estimated activation energy gave a value of 18,4 kJ/mol. All these facts are fully discussed. The rate of the reaction based on shrinking core model can be expressed by a semi-empirical equation as:

$$
\left[1-\left(1-X_{B}\right)^{\frac{1}{3}}\right]=\frac{12784 e^{\frac{-18400}{R T}} C_{A L 0}^{1,4686}}{\boldsymbol{\rho}_{B} R_{0}} t+0,1012 ; t \geq 1 \mathrm{~min} .
$$




\section{Introducción}

La principal fuente de zinc ha sido la extracción a partir de minerales y de subproductos industriales; residuos industriales de zinc y minerales de zinc como el sulfuro han sido utilizados en forma generalizada ${ }^{(1,2) ;}$ sin embargo, otros minerales como carbonatos ${ }^{(3)}$, los óxidos ${ }^{(4)}$ y los silicatos están reemplazando a los sulfuros debido a su escasez.

Es muy importante determinar la cinética de extracción de esta reacción química heterogénea. Tres etapas principales del proceso podrían controlar el mecanismo: difusión en la capa de fluido, difusión en el producto sólido y reacción química en la superficie. ${ }^{(1,6)}$.

Si la velocidad de reacción es controlada por la difusión en la capa de fluido, será aplicada la siguiente ecuación: $1-(1-X)^{\frac{2}{3}}=k_{d} y t$ en el

caso de control de la reacción química en la superficie: $1-(1-X)^{\frac{1}{3}}=k_{r} t(1)$ donde: $X$ representa la fracción de conversión del sólido y k es una seudo constante cinética, que indirectamente depende del orden de reacción en la concentración inicial del ácido, del tamaño promedio de las partículas y de la verdadera constante cinética de la reacción.

Este modelo es conocido como el "modelo del núcleo que se encoje sin reaccionar", que implica que la reacción se produzca en la superficie de la partícula y que su diámetro disminuye uniformemente en el transcurso de la reacción (11). Las muestras de sólido se utilizan clasificadas granulométricamente. Partículas esféricas es la forma más confiable para ajustar al modelo, y si el sólido está conformado por partículas de formas irregulares, la forma esférica puede considerarse como una buena aproximación que parece funciona bastante bien.

Si la energía de activación en esta reacción heterogénea es menor a $20 \mathrm{~kJ} / \mathrm{mol}$, se concluye que la etapa que controla el proceso es la difusión (trasporte de masa) y si es mayor a este valor, el proceso estará controlado por la reacción química, sin embargo, se observa datos controvertidos en la referencia. ${ }^{(3)}$.

En este contexto, se presenta el estudio de la cinética de extracción de las cenizas de zinc provenientes del proceso de galvanizado en caliente con ácido clorhídrico. La composición de las cenizas es compleja y el objetivo de este trabajo es aproximar a su cinética y el modelo.

\section{Parte experimental}

\section{Reactivos}

El ácido clorhídrico fue suministrado por Quimpac de Ecuador, sin ninguna purificación adicional.

Muestreo: Las cenizas de zinc se obtuvieron de Ideal Alambrec S.A., Quito, siguiendo un protocolo previamente establecido en normas 002-SA-2014.

\section{Técnicas}

Los diámetros de las partículas se midieron en un equipo CAMSIZER, para tres fracciones clasificaciones granulométricas que dieron valores de diámetro promedio 0,78 mm, 2,44 mm y $4,52 \mathrm{~mm}$.

Los metales se cuantificaron utilizando un equipo de Absorción Atómica, Perkim Elmer Analyzer 200, obteniéndose para estas tres fracciones los siguientes contenidos: Al $(0,12,0,21,0,11 \%)$, Fe $(0,82,1,17,1,157 \%)$.
La porosidad y la densidad se determinaron mediante técnicas comunes bien conocidas que utilizan picnómetro. La porosidad de las fracciones dio $(0,54,0,67,0,69)$.

Se utilizó la técnica de difracción de rayos X para determinar la composición de las cenizas. El equipo usado fue Bruker AXS D8 ADVANCE.

La reacción química se realizó en un reactor de vidrio de 5 litros de capacidad, provisto de chaqueta de refrigeración y sistema de agitación. Las determinaciones cinéticas se realizaron tomando muestras de líquido a intervalos de tiempo y midiendo la concentración de ácido clorhídrico por un método estándar normalizado. Al inicio de la reacción se mezclaron al ácido y el residuo sólido en proporciones estequiométricas.

\section{Resultados y discusión}

\section{El residuo sólido}

Las cenizas de zinc son un material heterogéneo y poroso compuesto principalmente por óxido de zinc y Zn, como se puede ver en la tabla 1. El difractograma de rayos X (ver figura 1), revela la composición de la tabla 1.

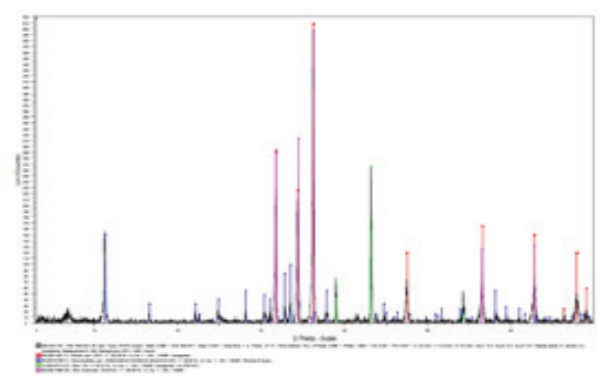

Figura 1: Difractograma de rayos $\mathrm{X}$ de las cenizas de zinc

Tabla 1

\section{Composición de las cenizas de zinc}

\begin{tabular}{|c|c|c|}
\hline Substancia & Fórmula & Contenido(\%) \\
\hline Zincita & $\mathrm{ZnO}$ & 49 \\
\hline Simonkolleita & $\mathrm{Zn} 5(\mathrm{OH}) 8 \mathrm{Cl} 2 \mathrm{H} 2 \mathrm{O}$ & 5 \\
\hline Zinc & $\mathrm{Zn}$ & 23 \\
\hline Zinc Hydroxide & $\mathrm{Zn}(\mathrm{OH}) 2$ & 23 \\
\hline
\end{tabular}

Las partículas presentan una morfología externa irregular (Figura 2) y su morfología interna (mononuclear, polinuclear, etc.) es desconocida. Esto implica que la distribución de zinc y su óxido es incierto y el material puede ser considerado como no homogéneo, lo que podría tener una influencia en la cinética de la reacción.

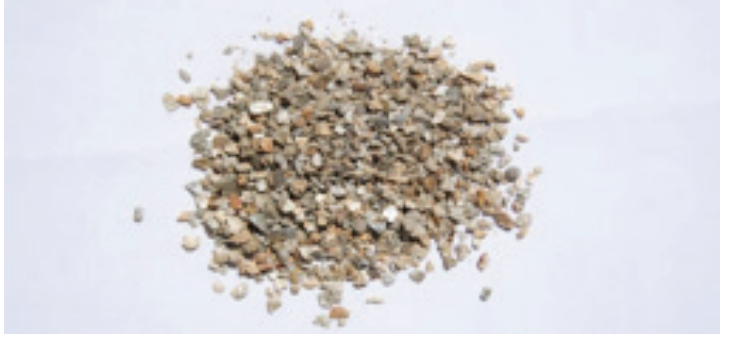

Figura 2: Fotografía de una muestra de partículas de diámetro promedio 4,52 mm 
La distribución de diámetros es simétrica (Figura 3), lo que indica que la contribución de los tamaños inferior y superior no es importante.

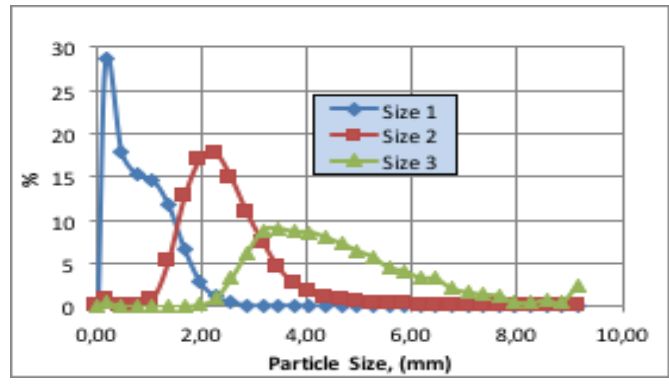

Figura 3: Distribución de tamaños del sólido

\section{Reacción con el ácido}

Las principales reacciones implicadas son las siguientes:

$$
\begin{gathered}
\mathrm{Zn}+2 \mathrm{HCl} \rightarrow \mathrm{ZnCl}_{2}+\mathrm{H}_{2} \\
\mathrm{ZnO}+2 \mathrm{HCl} \rightarrow \mathrm{ZnCl}_{2}+\mathrm{H}_{2} \mathrm{O}
\end{gathered}
$$

Una de ellas es una reacción redox, y la otra es un proceso de intercambio. Ambas reacciones contribuyen a la cinética global, sin embargo, la gráfica de consumo de ácido con el tiempo no presentó discontinuidades (Figura 4), lo que indica un proceso único, lo que ocurre a pesar de que la composición de las cenizas incluye un hidróxido conocido por su mayor reactividad con los ácidos.

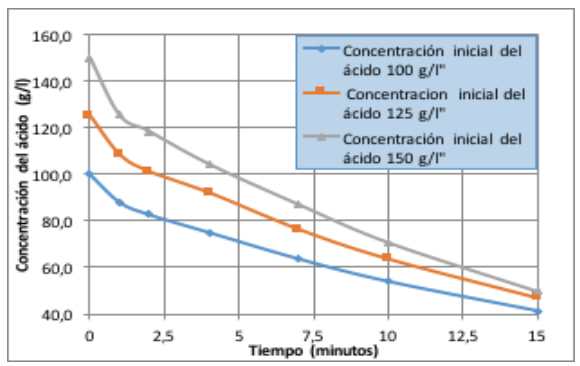

Figura 4: Influencia de la concentración inicial del ácido en el consumo de ácido durante la reacción. (Temperatura: $25^{\circ} \mathrm{C}$, Tamaño partícula 4,52 mm, agitación: $200 \mathrm{rmm}$ )

Este gráfico se aproxima a una curva exponencial y un primer periodo cinético resultó lineal hasta los 15 minutos (Figura 5), este es el orden interno. La principal condición para que el orden no se mantenga es que los parámetros están cambiando tal como la superficie del sólido que afecta a la velocidad.

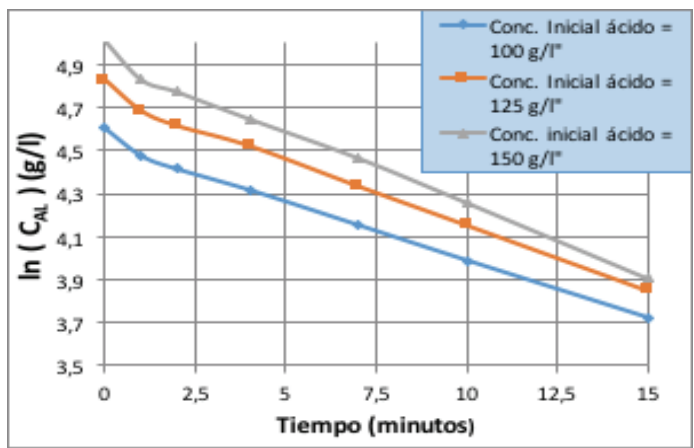

Figura 5: Primer orden de reacción respecto a la concentración de ácido. / (Tempe-ratura 25 C, Tamaño partícula 4,52 mm, agitación 200 rpm)

En la Figura 6. se muestra un gráfico log-log, de l a v elocidad de reacción frente a la concentración inicial que proporciona un orden externo o verdadero de 1,46. La discrepancia entre el orden interno y el orden externo se relaciona con el hecho de que los productos de la reacción pueden acelerar o disminuir la velocidad.

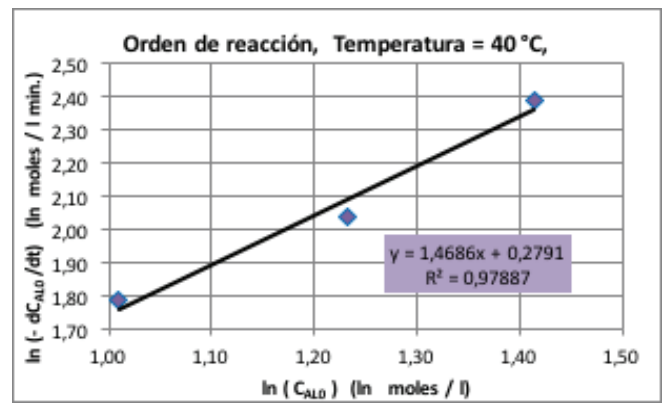

Figura 6: Orden de reacción desde gráfico log-log para consumo de ácido versus concentración inicial del ácido.

\section{Influencia del tamaño de las partículas}

Se utilizaron tres tamaños promedio de partículas $(0,78 \mathrm{~mm}, 2,44 \mathrm{~mm}$ y $4,52 \mathrm{~mm}$ ). La velocidad de reacción más alta dio para las partículas más pequeñas como era de esperarse en este tipo de reacción heterogénea. En la figura 7 se observa que la concentración del ácido cae rápidamente para el caso de $0,78 \mathrm{~mm}$.

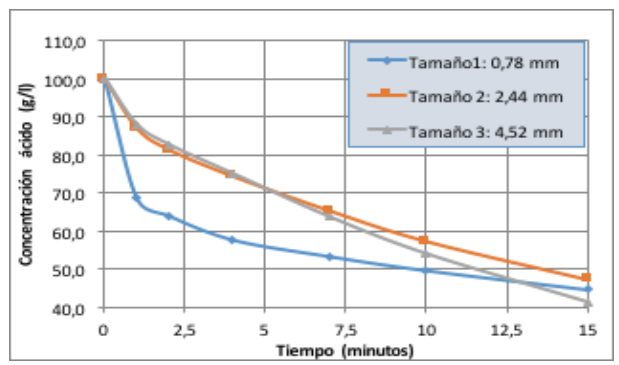

Figura 7: Efecto del tamaño de las partículas en la velocidad de reacción (Temperatura $25^{\circ} \mathrm{C}$, Concentración inicial ácido $100 \mathrm{~g} / \mathrm{l}$, agitación 200 rpm)

\section{Influencia de la velocidad de agitación}

Esta reacción a diferentes velocidades de agitación no presentó ningún cambio (Figura 8), que confirma que el proceso no está gobernado por el trasporte de masa.

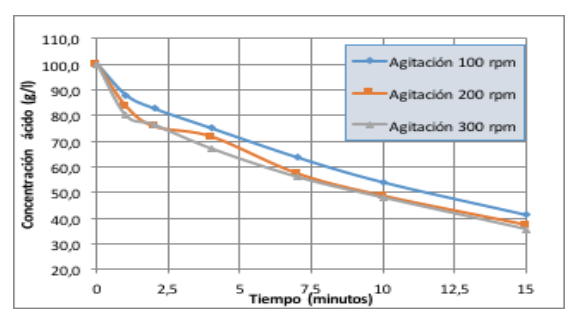

Figura 8: Influencia de la velocidad de agitación sobre la reacción.

(Temperatura $25^{\circ} \mathrm{C}$, concentración inicial ácido $100 \mathrm{~g} /$, tamaño partícula 4,52 mm)

\section{El modelo}

Varios modelos pueden estar involucrados en esta reacción heterogénea como se discutió en la introducción. El modelo del núcleo que se encoge sin reaccionar considerando como etapa controlante la reacción química implica una representación gráfica de ${ }_{(1}$ frengtę _a ${ }_{k_{r}}$ tiempo.

En la tabla 2 se observa que el ajuste a este modelo es bastante bueno. 
Tabla 2

Coeficientes de correlación para los modelos

\begin{tabular}{|c|c|c|c|c|c|c|c|}
\hline \multirow{3}{*}{$\begin{array}{l}\text { Modelo } \\
\text { Cinético }\end{array}$} & \multicolumn{6}{|c|}{ Coeficientes de Correlación } & \multirow{3}{*}{$\begin{array}{c}\text { Promedio } \\
\text { todas las } \\
\text { condiciones }\end{array}$} \\
\hline & \multirow{2}{*}{$\begin{array}{c}\text { Etapa } \\
\text { Controlante }\end{array}$} & \multirow{2}{*}{$\begin{array}{l}\text { Concentración } \\
\text { inicial del ácido } \\
\text { (g/l) }\end{array}$} & \multicolumn{4}{|c|}{ Temperatura $\left({ }^{\circ} \mathrm{C}\right)$} & \\
\hline & & & 25 & 30 & 35 & 40 & \\
\hline \multirow{3}{*}{$1-\left(1-X_{B}\right)^{2 / 3}$} & & 100 & 0,9948 & 0,9985 & 0,9901 & 0,9897 & \multirow{3}{*}{0,9881} \\
\hline & $\begin{array}{l}\text { Difusión en el } \\
\text { fluido }\end{array}$ & 125 & 0,9954 & 0,9805 & 0,9903 & 0,9848 & \\
\hline & & 150 & 0,9970 & 0,9769 & 0,988 & 0,9706 & \\
\hline \multirow{3}{*}{$1-\left(1-X_{B}\right)^{1 / 3}$} & & 100 & 0,9986 & 0,9994 & 0,9945 & 0,9956 & \multirow{3}{*}{0,9957} \\
\hline & Reacción Química & 125 & 0,9989 & 0,9906 & 0,9976 & 0,9964 & \\
\hline & & 150 & 0,9998 & 0,9895 & 0,9973 & 0,9901 & \\
\hline
\end{tabular}

El trazado gráfico para una concentración de ácido de $100 \mathrm{~g} / \mathrm{l}$ a cuatro temperaturas resultó lineal con buenos coeficientes de correlación (0,99), como se observa en la (Figura 9), sin embargo, las rectas se desvían del origen y presentan una ordenada. Se sugiere que al inicio el sistema no es completamente uniforme, teniendo un tiempo hasta estabilizarse posiblemente debido a varios factores, como la morfología, la composición compleja del sólido que incluye un hidróxido con una alta velocidad de reacción a los ácidos.

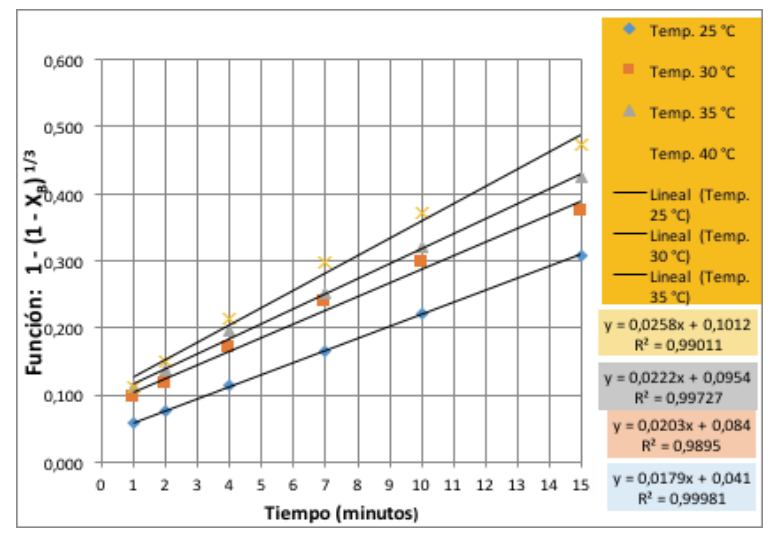

Figura 9: Trazado para el modelo del núcleo que se encoge sin reaccionar con etapa controlante la reacción química a cuatro temperaturas. (Concentración inicial ácido: 100 g/l, tamaño 4,52 mm, agitación 200 rpm)

\section{La energía de activación}

La constante seudocinética $k_{r}$ del modelo puede ser evaluada a partir de las pendientes de la Figura 9 y con los datos experimentales (diámetro, concentración de ácido), con el fin de obtener la verdadera constante cinética, como se discutió en la introducción.

Un gráfico de Arrhenius de la constante cinética versus 1/T, se presenta en la (Figura 10)

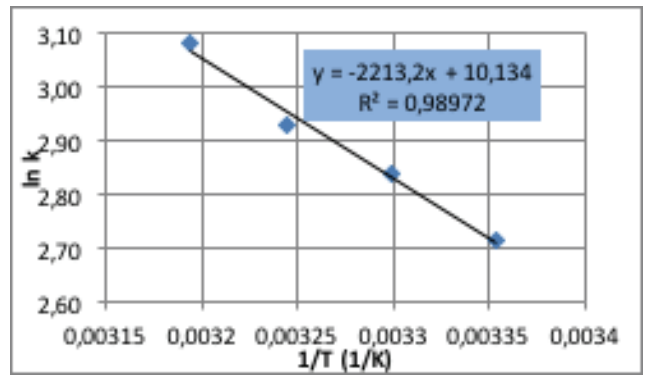

Figura 10: Gráfica de Arrhenius para el Modelo

La energía de activación calculada es 19,40 kJ/mol. Este valor sugiere que el proceso es controlado por la reacción química, según la literatura ${ }^{(1)}$.

\section{Conclusiones}

1. La reacción de ácido clorhídrico con cenizas de zinc residuo del proceso de galvanizado en caliente, está gobernado por un orden externo de reacción de 1,46 con una energía de activación de 19,40 kJ/mol.

2. La etapa controlante del proceso de recuperación de zinc de las cenizas con ácido clorhídrico, es la reacción química y no el trasporte de masa.

3. El modelo que mejor se ajusta para describir el comportamiento de la reacción es el del núcleo que se encoje sin reaccionar.

4. La cinética de la reacción se describe mediante la siguiente ecuación semi-empírica:

$$
\left[1-\left(1-X_{B}\right)^{\frac{1}{3}}\right]=\frac{12784 e^{\frac{-18400}{R T}} C_{A L 0}^{1,4686}}{\rho_{B} R_{0}} t+0,1012 ; t \geq 1 \mathrm{~min} .
$$

\section{Conflicto de intereses}

Los autores declaran no poseer conflicto de intereses para la publicación del presente artículo.

\section{Referencias bibliográficas}

- $\quad$ Bodas, M.G. (1996). Hydrometallurgical treatment of zinc silicate ore from Thailand,

- Dhawan, N., et al., (2011).Kinetics of hydrochloric acid leaching of smithsonite, Russian

- $\quad$ Feng, L., et al. (2007) Pelletizing and alkaline leaching of powdery low grade zinc oxide ores, Hydrometallurgy. 89, №. 3-4, 305-310.

- $\quad$ He, S., et al. (2010). Pressure leaching of high silica Pb-Zn oxide ore in sulfuric acid medium, Hydrometallurgy. 104, No. 2, 235240.

- $\quad$ Hollagh, A., et al. (2013). Kinetic Analysis of Isothermal Leaching of Zinc from Zinc Plant. Residue. International Journal of Nonferrous Metallurgy, 2, № 1, 10-20.

- $\quad$ Hydrometallurgy. 40, №. 1-2, 37-49.

- $\quad$ Hydrometallurgy. 89, №. 337-345.

- $\quad$ Hydrometallurgy. 105, No. 1-2, 186-190, (2010).

- Journal of Non-Ferrous Metals. 52, No. 3, 209-216.

- $\quad$ Langová, Š. et al. (2009). Selective leaching of zinc from zinc ferrite with hydrochloric acid, Hydrometallurg. y, 95, №. 3-4, 179-182.

- $\quad$ Levenspiel, 0. (2004). Ingeniería de las reacciones químicas. 3ra. Edición, México.

- $\quad$ Sethurajan, M., et al. (2016). Leaching and selective zinc recovery from acidic leachates of zinc metallurgical leach residues. Journal of Hazardous Materials.

- Souza, A.D., et al. (2007). Kinetics of sulphuric acid leaching of a zinc silicate calcine,

- $\quad$ Xu, H., et al., (2010) Sulfuric acid leaching of zinc silicate ore under pressure,

- Yoshida, T. (2003). Leaching of zinc oxide in acidic solution, Materials Transactions. 44, №. 12, 2489-2493. 